\title{
Psychiatric Diagnoses in a Sample of Outpatient Psycho-Geriatric New Referrals with Suspected Mild Cognitive Impairment
}

\author{
Jeremia Heinik $^{*}{ }^{, 1,2}$, Perla Werner ${ }^{3}$ and Gitit Kavé ${ }^{4,5}$ \\ ${ }^{I}$ Margoletz Psychogeriatric Center, Ichilov Hospital, Tel Aviv, Israel \\ ${ }^{2}$ Sackler Faculty of Medicine, Tel Aviv University, Tel Aviv, Israel \\ ${ }^{3}$ Department of Gerontology, University of Haifa, Haifa, Israel \\ ${ }^{4}$ Margoletz Psychogeriatric Center, Ichilov Hospital, Tel Aviv, Israel \\ ${ }^{5}$ Herczeg Institute on Aging, Tel Aviv University, Tel Aviv, Israel
}

\begin{abstract}
Psychiatric symptoms/syndromes are frequent in Mild Cognitive Impairment (MCI). However, only a few studies reported full psychiatric diagnoses in MCI. We describe the nosology and prevalence of psychiatric diagnoses in a group of 102 consecutive patients evaluated for suspected MCI and finally re-classified into dementia, MCI and No Cognitive Impairment. Psychiatric diagnoses were frequent in MCI and the other groups as well, however they were qualitatively different in each group.
\end{abstract}

Keywords: DSM-IV, psychiatric diagnoses, mild cognitive impairment (MCI), dementia, outpatient psycho-geriatric setting.

\section{INTRODUCTION}

The term mild cognitive impairment (MCI) is used to describe a group of elderly subjects who have cognitive impairments, often involving memory, not of sufficient severity to warrant the diagnosis of dementia [1]. Psychiatric disturbances are common in MCI $[2,3]$ even though they do not constitute part of its formal definition that emphasizes self and/or informant cognitive complaints, impairment in performance of objective cognitive tasks and essentially preserved activities of daily living $[4,5]$. They might affect etiologic considerations, clinical presentation, outcome, and treatment. With the aid of psychiatric and neuropsychiatric rating scales the prevalence of symptoms, symptom-groups and syndromes could be established in community and clinical samples. Psychiatric symptoms in MCI are estimated to range between 36.2 and 86.8 percent [6-9], depending on the various definitions and methodologies for assessment. However, only a few studies $[3,10,11]$ attempted to apply, even in part, official psychiatric diagnoses to their study samples of MCI and other subjects. In a population-based study, Forsell et al. [10] used DSM-III-R [12] criteria for the diagnosis of depression (with no further specification regarding this diagnosis), as well as a list of psychotic and anxiety symptoms derived from the above manual. In a clinical setting, Gabryelewicz et al. [11] defined major and minor depressive episodes according to DSM-IV criteria [13]. They excluded some individuals with severe major depression because these may have contributed to observed cognitive difficulties. In addition, they provided no specific definition and classification of depressive episodes, and reported only depression but no other types of psychopathology. Similarly,

\footnotetext{
*Address correspondence to this author at the Margoletz Psychogeriatric Center, Ichilov Hospital, 6 Weizman Street, Tel Aviv, Israel 64239; Tel: 972-3-6973325; Fax: 972-3-6974658; E-mail: heinik@post.tau.ac.il
}

in a sample of dementia clinic referrals Lopez et al. [3] used DSM-IV criteria for major depression but provided no further specification of their diagnoses. Thus far, only Kumar et al. [14] used specifically evaluated DSM-IV criteria for major and minor depressive syndromes in a small sample of community-dwelling, relatively young (60-64) subjects. Importantly, however, current nomenclature deals with disorders rather than syndromes.

The present study aims to describe the nosology and prevalence of DSM-IV axis I non-cognitive diagnoses in individuals referred for further evaluation for suspected MCI in the context of an outpatient psycho-geriatric setting, with special reference to the psychiatric diagnoses in those individuals finally diagnosed with MCI.

\section{MATERIALS AND METHODS}

The patient population referred to our outpatient psychogeriatric service includes mainly patients with cognitive impairment and individuals with depressive and anxiety disorders, treatable on an ambulatory basis. Each new referral undergoes a comprehensive multidisciplinary assessment process (geriatric psychiatrist, geriatrician, social worker, nurse). Laboratory investigations, including imaging studies, are suggested in each case to exclude potentially treatable causes for cognitive impairment and physical causes for emotional disorders. For those subjects with very early or early stages of cognitive impairment an MCI clinic was established, where subjects with a working diagnosis of suspected MCI undergo further evaluation. Subjects for the present study were recruited from these clinic referrals. The cognitive states of subjects with suspected MCI were further evaluated with the Hebrew version of the Cambridge Cognitive Examination - Revised (CAMCOG-R) $[15,16]$ (range $0-105 ; 80 / 81$ published cut-off point for cognitive impairment [15]), out of which an MMSE score can be generated 
(range $0-30 ; 23 / 24$ published cut-off point for cognitive impairment [17]). Psychiatric data were obtained with a modified Hebrew version of CAMDEX-R. The Cambridge examination for mental disorders of the elderly-revised (Heinik et al., in preparation), sections A (interview with patient), C (interviewer observations) and $\mathrm{H}$ (interview with informant). CAMDEX-R items makes it possible to derive various DSM-IV diagnoses [15]. This entire workup is usually completed within 1-2 months. The study protocol was approved by the local Helsinki committee.

For the purposes of the present study, DSM-IV [13] axis I operational criteria were used for cognitive and psychiatric diagnoses. When inapplicable, the criteria included in the DSM-IV "criteria sets and axes provided for further study" were used (e.g., minor depressive disorder, mixed anxietydepressive disorder). MCI diagnosis was established according to the Winblad et al. [5] criteria operationalized in the following order: 1. Not demented according to DSM-IV criteria, 2. Self and/or informant report of cognitive decline. 3. Impairment on objective cognitive task (defined as CAMCOG-R total score below the $25^{\text {th }}$ percentile compared to normative values by age-group, sex and educational level [18]. 3. Preserved basic activities of daily living/ minimal impairment in complex instrumental functions. 4. Not normal. Consensus regarding diagnosis was reached by two geriatric psychiatrists.

A total of 102 consecutive persons with suspected MCI were assessed. The overall mean age of the sample was 76 years $(\mathrm{SD}=6.3)$, and the mean years of education was 12
$(\mathrm{SD}=3.3)$. Females constituted $50 \%$. Final cognitive diagnoses were: dementia - 49 individuals (48\%; of which $63 \%$ with Alzheimer's type, $8 \%$ with vascular dementia, $10 \%$ with mixed type, $19 \%$ with other type dementia), mild cognitive impairment (MCI) - 36 individuals (35\%) and no cognitive impairment (NCI) - 17 individuals (17\%). Mean MMSE score in the total group was $26.37(\mathrm{SD}=2.27)$, and 24.81 $(\mathrm{SD}=1.79)$ in the dementia group, $27.6(\mathrm{SD}=1.76)$ in the MCI group, $28.12(\mathrm{SD}=1.36)$ in the NCI group. The mean total CAMCOG-R scores in the total sample were 82.73 (SD =9.36), with $76.31(\mathrm{SD}=7.96)$ in the dementia group, 86.25 $(\mathrm{SD}=5.38)$ in the MCI group, and $93.41(\mathrm{SD}=5.04)$ in the NCI group. The one-way Analyses of Variance (ANOVA) revealed significant group effects on the MMSE score (F $(2)=37.8, p<0.001)$ and the CAMCOG-R total score (F $(2)=48.6, p<0.001)$.

\section{RESULTS}

Table 1 shows that the total sample was almost equally divided into persons who received a psychiatric diagnosis or demonstrated DSM-IV predominant psychiatric features in dementia, and persons who had no psychiatric diagnosis. Most individuals within the dementia group did not receive any additional psychiatric functional diagnosis, and predominant psychiatric features (mostly depressive with or without anxiety) were found in under half $(43 \%)$ of this group. In the MCI and NCI groups, DSM-IV psychiatric diagnoses were found in $61 \%$ and $59 \%$ of the sample, respectively. Within the MCI group, categories suggested for possible inclusion in DSM-IV, such as minor depressive

\section{Table 1. Psychiatric Diagnoses and Predominant Features}

\begin{tabular}{|c|c|c|c|c|}
\hline & Dementia $(n=49)$ & $\operatorname{MCI}(n=36)$ & $\operatorname{NCI}(n=17)$ & Total $(n=102)$ \\
\hline No psychiatric diagnosis & $28(57 \%)^{\mathrm{a}}$ & $14(39 \%)$ & $7(41 \%)$ & $49(48 \%)$ \\
\hline Total psychiatric diagnoses & $21(43 \%)$ & $22(61 \%)$ & $10(59 \%)^{b}$ & $53(52 \%)$ \\
\hline Major depressive disorder (MDD) & - & $5(22.7 \%)^{*}$ & $3(30 \%)^{*}$ & $8(15 \%)^{*}$ \\
\hline Dysthymic disorder & - & $2(9 \%)^{*}$ & - & $2(3.7 \%)^{*}$ \\
\hline Bipolar disorder & - & $1(4.5 \%)^{*}$ & - & $1(1.9 \%)^{*}$ \\
\hline Post traumatic stress disorder (PTSD) & - & $1(4.5 \%)^{*}$ & $1(10 \%)^{*}$ & $2(3.7 \%)^{*}$ \\
\hline Generalized anxiety disorder & - & - & $1(10 \%)^{*}$ & $1(1.9 \%)^{*}$ \\
\hline Anxiety disorder NOS & - & $1(4.5 \%)^{*}$ & $1(10 \%)^{*}$ & $2(3.7 \%)^{*}$ \\
\hline Adjustment disorders $^{\mathrm{c}}$ & - & $1(4.5 \%)^{*}$ & $5(50 \%)^{*}$ & $6(11.3 \%)^{*}$ \\
\hline Minor depressive disorder & - & $5(22.7)^{*}$ & - & $5(9.4 \%)^{*}$ \\
\hline Mixed anxiety-depressive disorder & - & $6(27.2 \%)^{*}$ & - & $6(11.3 \%)^{*}$ \\
\hline \multicolumn{5}{|c|}{ Dementia predominant psychiatric features: } \\
\hline With delusions & $2(9.5 \%)^{*}$ & - & - & $2(3.7 \%) *$ \\
\hline With depressed mood & $15(71.4 \%)^{* d}$ & - & - & $15(28 \%)^{*}$ \\
\hline With behavioral disturbance & $4(19 \%)^{*}$ & - & - & $4(7.5 \%)^{*}$ \\
\hline
\end{tabular}

Abbreviations

MCI-Mild cognitive impairment; NCI-No cognitive impairment; NOS-Not otherwise specified. Dash indicates the absence of a specific psychiatric diagnosis/feature.

*Out of total psychiatric diagnoses in each diagnostic group.

Notes

${ }^{a}$ Denotes uncomplicated dementia.

${ }^{\mathrm{b}}$ One person had two psychiatric diagnoses (adjustment disorder and PTSD).

${ }^{\mathrm{c}}$ With the following subtypes: MCI - 1 with both depression and anxiety; NCI -3 with depression, 1 with anxiety, 1 with both.

${ }^{\mathrm{d}}$ Includes 4 persons with predominant depression and anxiety and 2 with predominant anxiety. DSM-IV offers no separate classification for dementia and anxiety, hence included under depression. 
disorder and mixed anxiety-depressive disorder accounted for $50 \%$ of psychiatric diagnoses. This group included 1 person with Major Depressive Disorder (MDD) with psychosis in partial remission and 1 person with bipolar disorder in remission. Within the NCI group, adjustment disorders prevailed. MDD was almost equal in frequency in the MCI and NCI groups. Thus, diagnoses suggesting psychosis and behavioral disturbance were noted in a minority of individuals in our MCI sample compared with the dementia group $(4.5 \%$ vs 28.6\%). In the MCI group minor mood disorders (dysthymic, adjustment, minor depressive and mixed-anxiety depressive disorders) were more common than major depres$\operatorname{sion}(63.4 \%$ vs $22.7 \%)$.

\section{DISCUSSION}

We investigated the nosology and prevalence of psychiatric diagnoses in a sample of subjects with suspected MCI seen at a specialized outpatient psycho-geriatric setting. The sample in total demonstrated reasonable performance on the cognitive tasks (mean scores for both tests above the published cut-off points for dementia), hence a suspected MCI diagnosis. However, a second in-depth evaluation led to reclassification of the original working diagnosis into dementia, MCI and NCI groups.

Psychiatric diagnoses were frequent in all three groups and highest in the MCI group (61\%). This finding is congruent with the significant amount of psychiatric symptoms/syndromes reported in this disorder [2, 3, 6-11,14]. However, the nature of the psychiatric diagnoses was qualitatively different in the three groups. In the NCI group the psychiatric diagnoses were minor traditional diagnoses (e.g., mostly adjustment disorders which might explain some diagnostic shifts during the diagnostic work-up from suspected MCI to NCI). In the MCI group suggested new minor diagnoses were significant, whereas in the dementia group predominant psychiatric features were found, though they did not amount to a full functional psychiatric diagnosis. It has been suggested $[8,19]$ that neuropsychiatric symptoms in MCI are quantitatively and qualitatively intermediate to that of healthy participants and those with dementia, and are therefore reminiscent of the clinical, neuroimaging and neuropsychological profile of MCI [19]. However this statement is true with regard to dementia of the Alzheimer type, amnestic MCI, and controls who are essentially free of any disturbances whatsoever. This is not the situation concerning our sample in which dementia etiology was heterogeneous and the NCI group was defined on the basis of cognitive functioning rather than on the exclusion of other disorders. Thus, we cannot commit as for the "intermediacy" of the MCI group concerning the psychiatric diagnoses.

Our findings concerning the low prevalence of diagnoses suggesting psychosis in MCI are in accordance with Chan et al. [2] and with Lopez et al. [3], both studies having demonstrated lower prevalence of psychosis and disruptive behavior in MCI compared with dementia patients, as well as with Lyketsos et al. [8] and Geda et al. [19] who reported symptoms of psychosis (delusions, hallucinations) and of disruptive behavior to be significantly lower in MCI compared with mild Alzheimer disease patients, albeit higher than in normal controls [19] and in MCI compared with dementia [8]. Similarly, minor depression was reported to be more prevalent in MCI than major depression, both in a clinical sample $(26.5 \%$ vs $19.6 \%$ [11]) and in a community sample (17.2\% vs 3.4\% [14]), and this was the case in the present study as well (63.4\% vs 22.7\%). The above stands for the minor nature of the psychiatric diagnoses we found among MCI subjects.

As for limitations of this study: First, the number of subjects in the total sample and in each diagnostic group was relatively small; Second, the study population was biased toward those subjects and caregivers cooperative enough to undertake prolonged cognitive assessment, as well as to those subjects in which demographics (schooling, fluency in language), sensory (appropriate sight and hearing capacity), physical (motor ability), and psychiatric conditions, are favorable for inclusion in such an assessment; Third, the definition of cognitive impairment we used differs from other psychometric definitions of MCI in which more stringent cut-points were used (e.g., -1.5 SD [3], -1 SD, beneath the $16^{\text {th }}$ percentile [20]). Fourth, the use of the entire DSM-IV multi-axial assessment would have probably provided us with a more comprehensive view of subjects and their ailments.

Even-though these limitations preclude generalization of our findings, the current work nonetheless demonstrates that psychopathology, expressed in clinical diagnoses, is common, heterogeneous and mostly mild in MCI. This might have repercussions regarding the psychiatric treatment of MCI. In fact, psychiatric diagnoses more than mere symptoms/syndromes should be evaluated in order to provide proper management of the MCI patient. The relationship between psychiatric symptoms, syndromes and full diagnoses in MCI warrants further investigation.

\section{ACKNOWLEDGEMENT}

We are grateful to Mrs. Rena Kurs for her editorial assistance in preparation of the manuscript.

\section{REFERENCES}

[1] Lopez OL, Becker JT, Jagust WJ, et al. Neuropsychological characteristics of mild cognitive impairment subgroups. J Neurol Neurosurg Psychiat 2006; 77: 159-65.

[2] Chan D, Kasper JD, Black BS, Rabins PV. Prevalence and correlates of behavioral and psychiatric symptoms in communitydwelling elders with dementia or mild cognitive impairment: the Memory and Medical Care Study. Int J Geriatr Psychiat 2003; 18 : 174-82.

[3] Lopez OL, Becker JT, Sweet RA. Non-cognitive symptoms in mild cognitive impairment subjects. Neurocase 2005; 11: 65-71.

[4] Petersen RC, Smith GE, Waring SC, et al. Mild cognitive impairment: clinical characterization and outcome. Arch Neurol 1999; 56: 303-8.

[5] Winblad B, Palmer K, Kivipelto M, et al. Mild cognitive impairment - beyond controversies, towards a consensus: report of the International Working Group on Mild Cognitive Impairment. J Int Med 2004; 256: 240-6.

[6] Palmer K, Berger AK, Monastero R, et al. Predictors of progression from mild cognitive impairment to Alzheimer disease. Neurology 2007; 68; 1596-602.

[7] Feldman H, Sceltens P, Scarpini E, et al. Behavioral symptoms in mild cognitive impairment. Neurology 2004; 62: 1199-201.

[8] Lyketsos CG, Lopez O, Jones B, et al. Prevalence of neuropsychiatric symptoms in dementia and mild cognitive impairment. Results from the Cardiovascular Health Study. JAMA 2002; 288: 1475-83.

[9] Ellison JM, Harper DG, Berlow Y, Zeranski L. Beyond the "c" in MCI: noncognitive symptoms in amnestic and non-amnestic Mild Cognitive Impairment. CNS Spectr 2008; 13: 66-72. 
[10] Forsell Y. Palmer K, Fratiglioni L. Psychiatric symptoms/syndromes in elderly persons with mild cognitive impairment. Data from a crosssectional study. Acta Neurol Scand 2003; 107 (Suppl 179): 25-8.

[11] Gabryelewicz T, Styczynska M, Pfeffer A, et al. Prevalence of major and minor depression in elderly persons with mild cognitive impairment - MADRS factor analysis. Int J Geriatr Psychiat 2004; 19: $1168-72$

[12] American Psychiatric Association. Diagnostic and Statistical Manual of Mental Disorders. Third Edition - Revised. Washington, DC: American Psychiatric Association, 1987.

[13] American Psychiatric Association. Diagnostic and Statistical Manual of Mental Disorders. Fourth Edition. Washington, DC: American Psychiatric Association, 1994.

[14] Kumar R, Jorm AF, Parslow RA, Sachdev PS. Depression in mild cognitive impairment in a community sample of individuals 60-64 years old. Int Psychogeriatr 2006;18: 471-80.
[15] Roth M, Huppert FA, Mountjoy CQ, Tym E. CAMDEX-R. The Cambridge examination for mental disorders of the elderly - revised. Cambridge, UK: Cambridge University Press, 1998.

[16] Heinik J, Werner P, Mendel A, et al. The Cambridge Cognitive Examination (CAMCOG): validation of the Hebrew version in elderly demented patients. Int J Geriatr Psychiat 1999; 14: 1006-13.

[17] Tombaugh TN, McIntyre NJ. The mini-mental state examination: a comprehensive review. J Am Geriatr Soc 1992; 40: 922-35.

[18] Williams JG, Huppert FA, Matthews FE, et al. Performance and normative values of a concise neuropsychological test (CAMCOG) in an elderly population sample. Int J Geriatr Psychiat 2003; 18: 631-44.

[19] Geda YE, Smith GE, Knopman DS, et al. De novo genesis of neuropsychiatric symptoms in mild cognitive impairment (MCI). Int Psychogeriatr 2004; 16: 51-60.

[20] Stephan BCM, Mattheus FE, McKeith I, et al. Early cognitive change in the general population: How do different definitions work? J Am Geriatr Soc 2007; 55: 1534-40.

Received: March 25, 2008

(C) Heinik et al.; Licensee Bentham Open.

This is an open access article distributed under the terms of the Creative Commons Attribution License (http://creativecommons.org/license/by/2.5/), which permits unrestrictive use, distribution, and reproduction in any medium, provided the original work is properly cited. 